\title{
Achieving Durable Dry Macula in Diffuse Diabetic Macular Edema: A Meta-analysis
}

\section{Avinoam Ophir*}

Professor, Department of Ophthalmology, Wolfson Medical Center, Holon, Israel

*Corresponding Author: Avinoam Ophir, Professor, Department of Ophthalmology,

Wolfson Medical Center, Holon, Israel. E-mail: ophir45avi@gmail.com
Received: February 08, 2021

Published: February 26, 2021

(C) All rights are reserved by Avinoam Ophir.

\section{Abstract}

Enduring diabetic macular edema (DME) might injure foveal layers, which may lead to progressive deterioration of visual acuity (VA). The aim of DME therapy is therefore to improve current VA and to achieve early, complete and durable macular drying. Therapy of centrally-involved diffuse DME (DDME) when macular traction is undetected has hitherto remained the major challenge. This meta-analysis focuses on the achievements of these two targets in DDME. Monotherapy by repeated intravitreal anti-vascular endothelial growth factor (-VEGF) medications does not achieve, as a rule, a lasting outcome. Modified grid laser photocoagulation (mGLP) was found efficacious in only a relatively small group of eyes. While using time-domain optical coherence tomography (TD-OCT), delayed pars plana vitrectomy (PPV) for intractable DME usually elicited a variable or no improvement of VA. After more than a decade of numerous DME trials by intravitreal medications, the two leading international study groups called for additional investigations to determine if VA can be better maintained with different management approaches.

The advent of high-resolution OCT has enabled a preoperative foveal layers assessment. Using this, early-PPV in DDME treatment-naive eyes attained very high efficacy in improvement of VA and in complete, durable macular drying. These findings can be explained by the two novel DDME pathogeneses. In contrast, delayed PPV achieved an improved VA but often only reduced the edema, leaving residual edema that necessitated continuous treatments. mGLP was found durably efficacious for DDME in specified conditions. Early-PPV and mGLP are also the most cost-effective therapies for DDME. Limitations refer to the lack of a controlled study of early-PPV, which provides a durable outcome, vs. current medications, which provide only a transient response. Until then, the long-lasting, highly efficacious, objective (OCT) outcomes of early-PPV, or mGLP in specific conditions, may initially assist in therapeutic decisions at least to populations where budgets and availability of medications are constraint. These two therapeutic approaches here described being perhaps their best or only options for saving their sights.

Keywords: Diabetic Macular Edema; Extrafoveal Traction; 3D-OCT; Pars Plana Vitrectomy; Grid Laser Photocoagulation; Anti-VEGF

\section{Abbreviations}

DME: Diabetic Macular Edema; VA: Visual Acuity; MAs: Microaneurysms; DDME: Diffuse DME; mGLP: Modified Grid Laser Photocoagulation; OCT: Optical Coherence Tomography; TD-OCT: Time-Domain Optical Coherence Tomography; PPV: Pars Plana Vitrectomy; VFT: Vitreofoveal Traction; VMT: Vitreomacular Traction;
ERM: Epiretinal Membrane; BCVA: Best-Corrected Visual Acuity; -VEGF: Anti-Vascular Endothelial Growth Factor; DRCR.net: Diabetic Retinopathy Clinical Research Network; CMT: Central Macular Thickness; ETDRS: Early Treatment Diabetic Retinopathy Study; SD: Spectral-Domain OCT; ONH: Optic Nerve Head; TCA: Triamcinolone Acetonide; VRI: Vitreoretinal Interface; ILM: Inner Limiting 
Membrane; PVD: Posterior Vitreous Detachment; FA: Fluorescein Angiography; EZ: Ellipsoid Zone; ELM: External Limiting Membrane; DRIL: Disorganization of the Inner Retinal Layers; CNP: capillary Non-Perfusion; FAZ: Foveal Avascular Zone; SS OCT: Swept Source.

\section{Introduction}

In diabetic macular edema (DME), the longer the edema the greater visual acuity (VA) loss [1]. This loss is mainly related to progressive macular layers injury and to ischemic processes [2-4]. The ultimate aims of DME therapy are therefore to improve current VA and to achieve early, complete and durable dry macula.

Treatment of focal DME by focal laser photocoagulation to leaking microaneurysms (MAs) is well acceptable[4]. In diffuse DME (DDME), pars plana vitrectomy (PPV) to remove vitreofoveal traction (VFT; often termed "vitreomacular" - VMT), or associated with epiretinal membrane (ERM) removal when indicated, were commonly found durably efficacious [5,6]. Centrally involved DDME when VMT and ERM traction were undetected, commonly termed "non-tractional", has hitherto remained the major DME challenge [5]. This study presents and explains the cumulative data on the high efficacy of early-PPV for attaining durable and complete macular drying in DDME without detected traction, and of mGLP in specific conditions.

\section{Methods}

A search of all therapeutic, peer-reviewed meta-analysis and review articles published until January 2021 in English was conducted on the PubMed. Included in this meta-analysis are all PPV studies for DDME without detected traction, in which preoperative assessment of the central macular layers by high resolution OCT could approve surgery. The keywords used included focal and diffuse diabetic macular edema, focal laser, grid laser photocoagulation (GLP), pars plana vitrectomy, anti-vascular endothelial growth factor (-VEGF), bevacizumab, ranibizumab, aflibercept, steroids, diabetic epiretinal membrane (ERM), optical coherence tomography (OCT), OCT-angiography (OCTA), vitreomacular-, vitreofoveal- vitreopapillary- and extrafoveal traction, and vitreoretinal interface. This meta-analysis primarily referred to studies that involved DDME alone. Best- corrected visual acuity (BCVA) and complete and durable macular drying were the primary endpoints.
There is no risk of bias regarding the studies on PPV or mGLP in DDME when they present superiority over administration of intravitreal medications.

\section{Results and Discussion}

Intravitreal medications

During the last decade, anti-VEGF medications became the first choice of DME therapy [7-10]. The most currently used medications are ranibizumab (Lucentis, Genentech, San Francisco, CA), bevacizumab (Avastin, Genentech) and aflibercept (Eylea, Regeneron, Tarrytown, NY). These pivotal prospective randomized controlled studies on anti-VEGF or steroids medications, and thereafter, have typically merged focal and diffuse DME, as well as focal/grid laser photocoagulation, in one group, "DME", for calculations and conclusions. For example, in the pivotal 'Protocol I' study ( $\mathrm{n}=854$ eyes) by the Diabetic Retinopathy Clinical Research Network (DRCR.net), repeated, initially monthly intravitreal administrations of ranibizumab were accompanied by prompt or deferred focal/grid laser photocoagulation [7]. Outcomes were compared with focal/grid laser (+sham) alone as the control group (32-36\% had focal DME and 42-43\% had DDME). After 12 months, 8-9 injections combined with the laser therapies have reached a mean gain of 9 Early Treatment Diabetic Retinopathy Study (ETDRS) letters. At 24-months ( $n=275$ eyes), mean gain was 8 letters, BCVA improved by $5-\geq 15$ letters in $71 \%$ of eyes, and a loss of $\geq 15$ letters occurred in $2 \%$ [9].

However, following more than a decade of numerous trials on intravitreal medications for DME therapy, major drawbacks still exist: Current anti-VEGFs efficacies are short-lived, and when administered as monotherapy none, as a rule, achieves durable macular drying. Moreover, these therapies are guided by a trial-and-error approach, commonly involving switches among medications. Most anti-VEGFs studies used laser photocoagulation either as predetermined, or often as a trial-and-error rescue, with its consequences [7-10]. Therefore, these therapies are commonly administered for years. Moreover, most anti-VEGFs and steroid implants are costly; the former were deemed by the DRCR.net authors to be "not cost effective". The anti-VEGF regimen also entails a burdensome schedule of regular office visits to patients and personnel. Switching to treatments by the current intravitrealsteroids, triamcinolone acetonide (TCA), dexamethasone implant or fluocinolone acetonide, is commonly complicated by cataract and not infrequently by increased intraocular pressure necessitating hypotensive therapies. 
Moreover and importantly, substantially poorer outcomes of anti-VEGFs were generally reported in real world studies in comparison with the major prospective randomized controlled trials [11-13]. In the USA study surveying $>15,000$ patients, $>5100$ eyes were treated with ranibizumab for 24 months [11]. After 7.7 and 12.7 injections, the mean BCVA improved by 4.7 ETDRS letters at 12-months and reduced to only a 3.4-letter gain at 24- months, respectively. In eyes with a base-line BCVA of $\geq 6 / 12$, VA worsened by 2.4 and 3.4 letters at 12- and 24- months, respectively. The real-world UK study included 3,103 eyes across 19 hospitals and reported on only 3 (at least) ranibizumab injections per year [12]. After 2 years, $17.3 \%$ of eyes gained $\geq 15$ letters but as many as $23 \%$ of eyes lost one to $>30$ letters. In another real-world study, a database of aggregated electronic medical records of 28,658 eyes was analyzed [13]. At one year, eyes underwent a mean of 6.4 anti-VEGF injections; $50 \%$ of eyes received $\leq 6$ injections, while $<20 \%$ received 10-13 injections, representing monthly treatment. At one year, mean of +4.2 letters was gained, which generally showed a linear relationship with mean number of anti-VEGF injections. Eyes with good baseline VA $(\geq 6 / 12)$ generally were at risk of VA loss at one year. These outcomes are substantially inferior to mean gain of 9 or 8 letters in Protocol I study after 12 and 24 months, respectively $[7,9]$. These real-world outcomes highlight the concerns that probably millions of DME patients world-wide receive a significantly smaller number of injections than recommended by the large prospective studies, or even none at all, for reasons of costs and lack of treatment facilities.

Following more than a decade of numerous DME trials by intravitreal medications, a deadlock has been affirmed by the leading international study groups: The pivotal two-year DME Protocol-T of the DRCR.net $(n=660)$ compared outcomes of the three mostly used anti-VEGF medications, ranibizumab, aflibercept and bevacizumab [10]. Aided by focal/grid laser therapies as rescue in $\sim 45 \%$ of eyes, all three treatment-groups improved in VA. Following completion of the 2-year study, the study was extended to 3 more years, and 317 eligible participants were managed with standard, real world care [14]. At 5 years, the mean CMT had decreased from baseline by $154 \mu \mathrm{m}$, and was stable during the last 3 years $(-1 \mu \mathrm{m}$ difference). At 5 years, the mean BCVA had improved from baseline by 7.4 ETDRS letters, but decreased by 4.7 letters during the 3-year extension period; each medication-group was associated with substantial reduction in VA due to persistent or recurrent edema. At the conclusion of the study, the DRCR.net USA group affirmed
(2020) that "additional investigation into strategies to improve long-term outcomes in DME seems warranted, to determine if BCVA can be better maintained with different management approaches". Similarly, in the comprehensive EURETINA review guidelines for DME therapy (2017), this leading European group expressed their trust that "the most recent OCT technology with high resolution and dense scanning will help automation of 3-dimensional imaging analyses and detection of disease activity from small morphological changes early on" [4]. Indeed, the point-by-point OCT scans with high- resolution 3D spectral-domain (SD) OCT figures and video clips have fulfilled that hope. This approach enabled detection of overlooked extrafoveal traction membranes that are commonly associated with DDME [15-19].

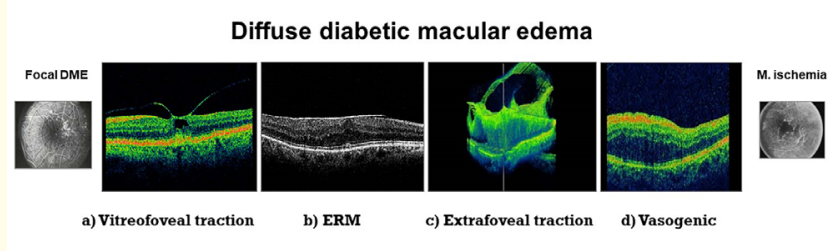

Figure 1: Optical coherence tomography (OCT) of focal and diffuse DME. Diffuse DME may be associated with,

a) vitreofoveal traction, b) tractional epiretinal membrane, c) extrafoveal traction, or d) vasogenic, non-tractional. Macular ischemia may be associated with each of these types.

\section{Two novel pathogeneses of diffuse DME}

Evaluation of DME by OCT is regularly performed by raster lines or 6-radial scanning, and typically centeredonly on the macula [710]. These approaches, however, leave most of the potential sites of extrafoveal traction among the scan lines, including the optic nerve head (ONH) site, unscanned (Figure 2).

A thorough TD-OCT-2 scanning search (Carl Zeiss Meditec, Germany) in DDME eyes enabled a b-scan detection of extrafoveal vitreous traction membranes in $10.8 \%(n=186)$ of eyes (Figure 3) [15]. The advent of 3D SD-OCT (Topcon 1000, Japan) enabled fast scanning and coverage of each point at the vitreoretinal examined fields, associated with 3D figures and video clips of extrafoveal traction membranes associated with DDME [16,18]; (Figure 4). A video clip and additional information are given in: link to the video. The $3 \mathrm{D}$ SD-OCT plainly enabled the detection 


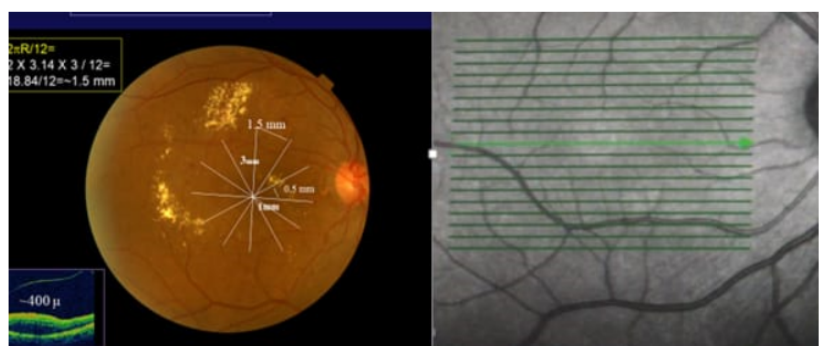

Figure 2: Standard OCT scanning in DME is regularly associated with large skipped areas. Using 6-radial lines (left figure), the distance between two lines at $3-\mathrm{mm}$ radius from the fovea is $>1,500 \mu(2 \times 3.14 \times$ radius $)$, and $>500 \mu$ at only $1 \mathrm{~mm}$ from the fovea. Similarly, using raster lines (right figure), most points in the examined field are unscanned. The skipped areas among the scan lines as well as other commonly unscanned fields at the area centralis, including the optic nerve head site, may result in missing most potential extrafoveal traction sites.

Bottom left (small figure): Diffuse macular edema, in which the posterior hyaloid is detected away from the edematous fovea.

This would appear when the scan line does not cross the contact site of the retina and posterior hypoid.

of two novel DDME pathogeneses (Figure 1-C,D) [16]. Their prevalence among DDME pathogeneses $(n=58)$ was (Figure 1$)$ : a) Extrafoveal vitreoretinal and/or vitreopapillary traction $-34.5 \%$ $(n=20)$ of eyes. This indicated that vitreous traction may emerge anywhere in the area centralis secondary to incomplete posterior vitreous detachment (PVD); b) vasogenic (true non-tractional) $-24.1 \%(\mathrm{n}=14)$, after unequivocal exclusion of traction, including extrafoveal; c) VFT -19.0\% ( $\mathrm{n}=11)$; and d) central ERM -22.4\% ( $\mathrm{n}$ $=13$ ) of eyes. The prevalence ration of the two novel pathogeneses (earlier considered a "non-tractional" group) was 59\% and $41 \%$ of extrafoveal traction vs. vasogenic DDME, respectively. Therefore, tractional pathogeneses comprise the majority of DDME eyes. The high efficacy of PPV in DDME eyes has been further discussed $[18,19]$. Based on the outcomes in the anti-VEGFs trials [7-10] we may assume that a partial and temporary edema reduction could be attained in eyes with overlooked extrafoveal tractional membranes and ERMs (ERMs were included in most of the major anti-VEGF studies [7-10]). This, by the anti-VEGF effect on the leaking capillaries. Overlooked extrafoveal traction may also explain the common failures of mGLP and even edema worsening in DDME, and itsdurable efficacy in most vasogenic DDME [17,20,21].

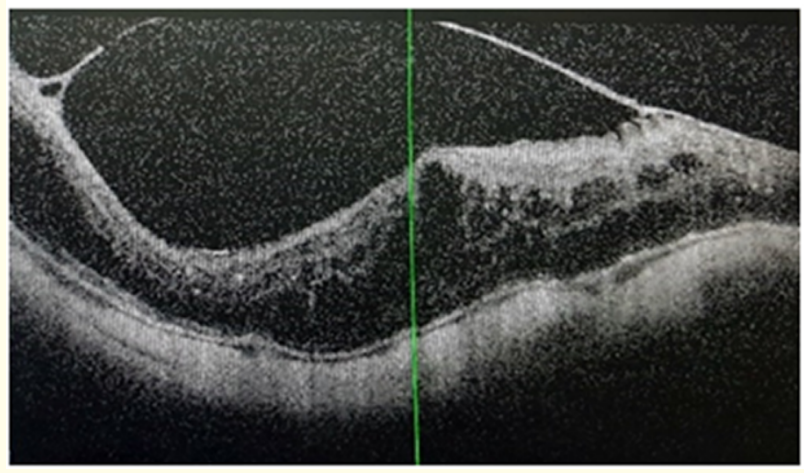

Figure 3: Verifying extrafoveal traction association with the DDME. A continuity between the retinal edema underlying the small traction site and the edematous fovea would validate their association. The anomalous, incompletely detached posterior hyaloid is in continuum with the ERM (right side).

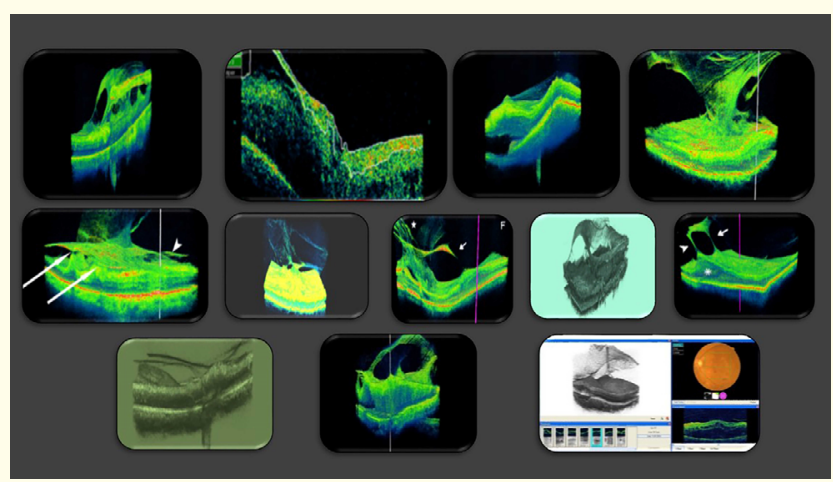

Figure 4: Extrafoveal traction in DDME. Vitreopapillary (upper row) and extrafoveal vitreoretinal traction (two lower rows), each is associated with foveal edema (vertical lines). The foveae are free from traction, except one eye (left lower) with combined vitreopapillary and vitreofoveal traction. 
Additional studies using 3D-OCT similarly detected extrafoveal traction membranes. Fatima., et al. also used3D SD-OCT (Topcon 1000 ) in a study that included focal and diffuse DME. Of the tractional cases $(\mathrm{n}=17)$, most $(65 \%)$ had extrafoveal traction [22]. Adhi., et al. used an en-face technique of Swept Source 3D OCT (Triton, Topcon), which holds a continuous scanning. They reported that 41\% (11/27) of DDME eyes were associated with retinal "adhesions/ pegs" [23]. Figures 2-4 in this article identify them as extrafoveal traction membranes. Additional images of extrafoveal traction in DDME were presented elsewhere [24].

\section{Vitreoretinal interface in diffuse DME}

The status of the posterior vitreous cortex is a major factor in DME emergence or cure. Except for its potential traction, the vitreous cortex in DME is rich in proangiogenic factors such as VEGF, cytokines, chemokines, oxygen reactive species and proinflammatory molecules, which promote a breakdown of theblood retinal barrier. PPV can reduce DME by removing these, as well as by increasing the oxygenation in ischemic retinal areas [25]. In the few PPV comparative studies, substantial superiority of PPV outcomes was found in eyes without detected traction over antiVEGF or TCA medications and/or mGLP [26,27].

In histological and immunocytochemistry studies, Gandorfer et al. and Hagenau et al. described the posterior vitreous cortex in DDME following PPV and inner limiting membrane (ILM) peeling ( $n=61$ and 27, respectively) [6,28]. They detected its remodeling associated with trans-differentiation of hyalocytes into contractile myofibroblasts, fibroblasts and fibrous astrocytes in most eyes. The contractile cells situated within the posterior vitreous at the vitreoretinal interface (VRI) may result in tangential vitreomacular traction. The authors claimed that these findings argue for an early surgical intervention in DDME irrespective of the presence of traction formation imaged by OCT [28].

Schepens' group (1993), Schwartz and associates (1996) and Sebag described a typical emergence of a split in the posterior vitreous cortex, "vitreoschisis", associated with incomplete, anomalous posterior vitreous detachment (PVD) in diabetic retinopathy [29]; (Figure 5). When the attached posterior leaf of the split, presented as ERM, is in continuum with the anteriorly detached leaf, it may result in centrifugal ERM traction resulting in DDME. Demonstrated in video clips, such unified ERM/posterior hyaloid membranes ("Evi" membrane) were detected in 20 of
$23(87 \%)$ consecutive ERM-related DDME eyes associated with incomplete, anomalous PVD [30]. Whereas PPV and ERM removal was found efficacious in ERM-associated DDME [6,31], a reduced response to ranibizumab and none or even worsening response to mGLP has been reported in such eyes [17]. In evaluating PPV outcomes as a rule, poor diabetic control, systemic hypertension, lipidemia and sub-foveal hard exudates may also be associated with thickened macula and worse VA.

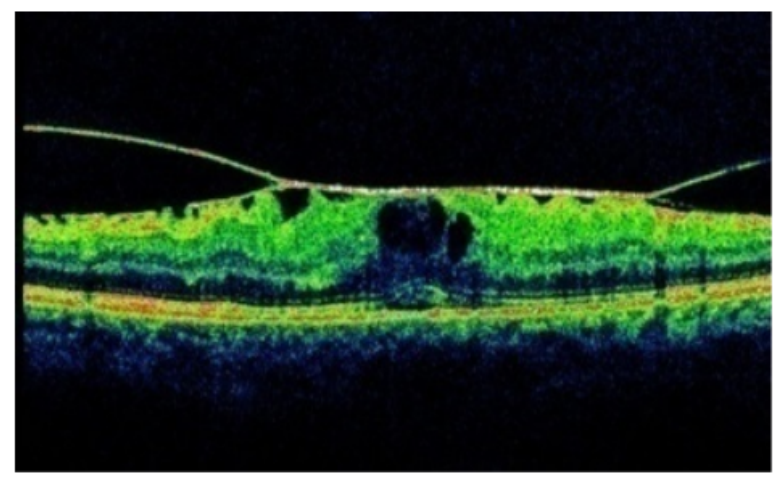

Figure 5: Vitreoschisis. A split in the posterior vitreous cortex presents the ERM, which is its attached posterior leaf. The ERM is in continuum with the anterior leaf, the detached posterior vitreous cortex ("Evi" membrane). This association may result in centrifugal ERM traction and diffuse macular edema.

\section{PPV outcomes in diffuse DME}

In 1992, before the OCT era, Lewis et al. were the first to report that PPV had been beneficial in 10 eyes with tractional DDME associated with taut, thickened and glistening premacular posterior hyaloid, detected ophthalmoscopically [32]; (Figure 6). High efficacy in similar studies, commonly associated with complete and durable resolution of the edema, diagnosed ophthalmoscopically or by fluorescein angiography (FA), and improved VA [33,34], encouraged researchers to perform PPV even in DDME eyes without detectable traction. Surgeries took place mostly following failed mGLP trials, and quite-often, though not always, resulted in long- lasting improvement of macular edema and VA in the majority of the eyes [5]. During the last decade, intravitreal antiVEGF medications and steroids became the gold standard for trials and treatments for DME [7-10]. 


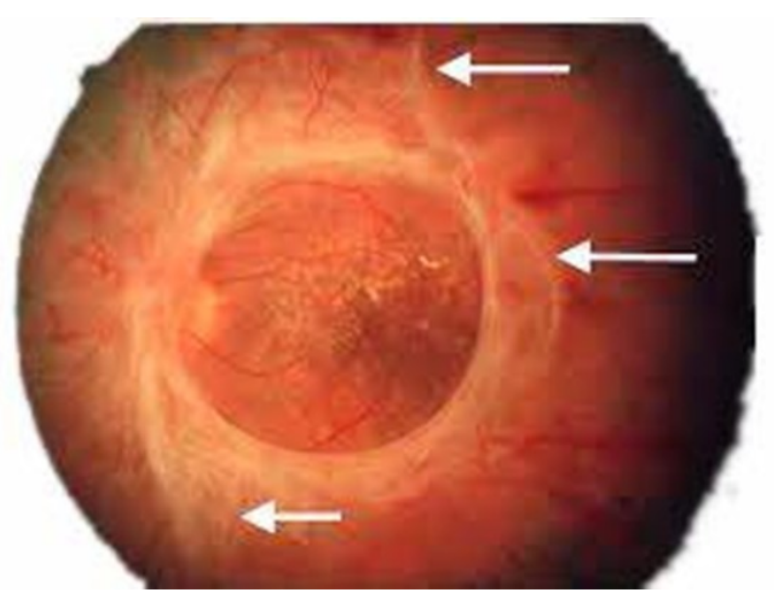

Figure 6: Diffuse DME associated with taut posterior hyaloid. The taut posterior hyaloid (arrows) is tenting the macular surface. Macular scars following modified grid laser photocoagulation are present.

Since these medications were commonly administered during many months or years, a substantially longer delay in conducting PPV surgery for the persistent edema naturally followed. When TD-OCT was used, which did not allow for assessing the integrity of external limiting membrane (ELM) and inner segment/outer segment junction (ellipsoid zone = EZ), PPV outcome was often associated with variable or even no improvement of VA. This topic has been thoroughly analyzed in various review and meta-analysis studies $[4,35]$. The mean low VA gain has caused ophthalmologists to further postpone PPV when DME had been resistant to non-surgical therapies. The enduring edema could result in further foveal layers injury.

The advent of SD-OCT with its faster scanning and higher resolution enabled an accurate preoperative assessment of the foveal layers integrity. It enabled improved prognosis of postoperative VA, which was unachievable by the TD-OCT $[4,35,36]$. Browning et al. concluded that sight-threatening complications (retinal detachment) following small-gauge vitrectomy may be similar to three-year risk of endophthalmitis (1-1.5\%) in eyes treated primarily with anti-VEGF injections [37]. The arguments for, or sometimes against ILM peeling have already been thoroughly reviewed.

PPV: Achieving durable macular drying and improved VA PPV for DDME associated with extrafoveal tractional prior to the OCT era

This group presents a high PPV efficacy in tractional DDME eyes, many of which were of the extrafoveal vitreous traction type.

- "Thickened, taut posterior hyaloid". These membranes have been described by few authors as "different from vitreomacular traction" and "tenting the macular surface" [33,34], i.e. extrafoveal traction membranes in many of them, as discussed [26]; (Figure 6). For example, PPV was carried out in 10 to 55 DDME such eyes, mostly after failed mGLP treatments [6,32-34]. Macular edema has completely dried in 50-98\% of eyes and improved in most others. Improved BCVA was attained in $47-92 \%$ of eyes after $\geq 12$ months (mean) of follow- up.

- Vitreopapillary traction. Durable success in macular drying and BCVA was reported in $83 \%$ of 17 eyes after 14.5 months (mean) of follow-up [38]. Explanation to outcome may be deduced from the 3D-OCT studies (Figure 4).

- Extrafoveal traction associated with diffuse macular edema and impairment of perfusion at the tractional area extending into the fovea [24]. The extrafoveal traction membranes were detected ophthalmoscopically as well as by wide-field OCT ( $n=2$, one with DDME). PPV achieved durable macular drying and improved VA, associated with resumption of perfusion. A discussion followed.

\section{Delayed PPV in intractable DME using TD-OCT}

Landers and associates [35] analyzed the DRCR.net PPV study on 115 (of 241) eyes without VRI abnormalities that had been intractable to intravitreal bevacizumab and/or corticosteroids and macular laser therapy [31]. These eyes were preoperatively considered by 74 surgeons unlikely to respond to further macular laser photocoagulation, i.e. assumingly having severely damaged maculae. More than $90 \%$ of operated eyes experienced improved CMT at six months postoperatively. However, mean VA did not improve. The researchers have mentioned that since the investigators had only TD-OCT images, and FA was not done preoperatively, they had no way of determining both the integrity of the foveal layers and the macular perfusion status [35]. 
Along with other review articles, the EURETINA group analyzed PPV outcomes in nine DME studies and Landers et al. reviewed 37 studies in several articles, all had mostly used TD-OCT [4,35]. Most studies had involved eyes intractable to non-surgical therapies, after excluding eyes with VFT and ERM. A combination of no-response to anti-VEGF medications and macular laser, long-delayed PPV and the use of TD-OCT was the rule in most of the reviewed studies. In these, surgery typically resulted in improved CMT, and in $\geq 2$ lines VA gain in $\sim 50 \%(0-90 \%)$ of eyes. The EURETINA group outlined the controversy on the benefit of PPV due to inconsistency in VA outcomes [4]. Similarly, conclusions based only on mean VA while using TD-OCT could be, in retrospect, inadequate [7-10].

\section{Using SD-OCT prior to PPV}

Perioperative integrity of central macular layers

Outcomes of all PPV found studies for DME, in which integrity of the central macular layers had been preoperatively assessed with high-resolution OCT, are presented in table 1 ( $\mathrm{n}=18$ to 120 eyes; the articles commonly used the term "DME", which included DDME). Eyes that had had extinct photoreceptors EZ and/ or ELM bands were not included. Excluded from the Table were also studies, a) that had mainly involved eyes with proliferative diabetic retinopathy, due to the potential effect of vitreous hemorrhage removal on final VA (Shah 2012); b) in which only the eyes with postoperative complete DME resolution had been evaluated. For example, Yanyali examined 11 eyes with completely dry maculae that had undergone PPV 87 months (mean) earlier [39].The higher the percentage of foveolar intact EZ/ ELM bands, the better was the final VA; c) that had vitreoretinal interface abnormalities (Hwang, 2021). All excluded studies had typically achieved similar PPV outcomes to those of Nos. 1-6 in table 1.

Four of the studies in table 1 (Nos. 2,3,5,7) concluded that the integrity of the EZ and/or ELM bands may be used as prognostic biomarkers of PPV in DME. Disorganization of the inner retinal layers (DRIL) is also considered a DME biomarker in macular injury [3]. In contrast, Uji (No. 1), Browning (No. 6) and Michalewska (No. 8)., et al. did not find an association between the preoperative degree of EZ/ELM injury and postoperative improvement in VA. When comparisons were made within studies, eyes that had had preoperative foveal layers integrity loss experienced lower percentage of postoperative visual improvement than eyes with intact foveal layers (Nos. 3,5,7).

\section{Delayed PPV using perioperative SD-OCT}

In all eight studies (Table 1), PPV and ILM peeling in eyes without detected traction (at least in most eyes) significantly resulted in improved VA and CMT. Six studies (Nos. 1-6, Table 1) reported on delayed PPV in eyes that have been most commonly refractory to intravitreal medications and laser therapies. These outcomes, and outcomes of the aforementioned excluded studies, underscore the potential of delayed PPV while using high-resolution OCT in achieving improved current VA. Complete macular drying was found in one-third to two-thirds of the eyes (reports from studies $2,4,6$ ), associated with mean reduction in the needs of postoperative therapies. However, there was no report whether FA was done postoperatively prior to initiating the supplementary treatments in these studies. Laser therapies to leaking capillaries and/or MAs could potentially achieve durable dry maculae, since these eyes turned postoperatively to have the vasogenic DME type (focal or diffuse). Moreover, studies have reported on significant associations between the degree of macular or peripheral capillary non-perfusion (CNP) areas, enlarged foveal avascular zone (FAZ), macular ischemia, CMT, large foveal cysts or DRIL and VA in DME [3,17]; (Figure 7). Therefore, FA (or OCT-angiography) could potentially provide another explanation of the residual or recurrent postoperative macular edema. The two other studies (Nos. 7,8, Table 1) reported on early-PPV in naïve-treatment eyes $[40,41]$. In these, surgery has reached $100 \%$ and $93 \%$, respectively, of durable completely dry maculae.

Early-PPV or PPV in DDME naïve-treatment eyes: high and durable success rates

A key condition for improved DME therapy is the timing of treatment since diagnosis. In a comparative study before the OCT era, La Haij et al. reported on PPV conducted between two to 36 months (mean, $\sim 11$ ) after edema diagnosis [42]. Naïve-treated eyes $(n=7)$ had a significantly higher percentage $(77 \%)$ of VA improvement than eyesfollowing laser treatment $(n=12 ; 14.8 \%)$. In a prospective PPV study by Yanyali et al. on naïve-treated DDME eyes using TD-OCT: 11 of 12 (92\%) eyes attained complete dry maculae six months postoperatively; BCVA improved by $\geq 2$ lines in six eyes (50\%) and remained stable in the others [27]. Using SD-OCT, a multi-national retrospective study was conducted by Iglicki et al. on 120 naïve-treatment DDME eyes within $\leq 12$ months 


\begin{tabular}{|c|c|c|c|c|c|c|}
\hline $\begin{array}{c}\text { Author } \\
\text { (Refer. No.) }\end{array}$ & $\begin{array}{c}\text { Naïve } \\
\text { Refractory }\end{array}$ & $\begin{array}{l}\text { PPV+ILM peeling; } \\
\text { Associated macular } \\
\text { laser/phaco/ERM }\end{array}$ & $\begin{array}{c}\text { Preop. foveal layers: } \\
\text { Predictive } \\
\text { Factors for postop. VA }\end{array}$ & $\begin{array}{l}\text { No. of eyes; } \\
\text { Follow-up } \\
\text { (months) }\end{array}$ & $\begin{array}{l}\text { Pre./ postop } \\
\text { logMAR VA } \\
\text { (mean) }\end{array}$ & $\begin{array}{l}\text { Pre./ postop } \\
\text { CMT }(\mu \mathrm{m}) \\
\text { (mean) }\end{array}$ \\
\hline 1. Uji (46) & $66 \%$-Refr. & $\begin{array}{c}\text { Phaco-73\%; PDR-31\%; } \\
\text { ERM-33\% }\end{array}$ & Parallelism & $64 ; 6 \mathrm{~m}$ & $0.5 / 0.35 \mathrm{~S}$ & $481 / 313 \mathrm{~S}$ \\
\hline $\begin{array}{l}\text { 2. Miyamoto } \\
(47)\end{array}$ & $50 \%$-Refr. & $\begin{array}{c}\text { All rendered pseudopha- } \\
\text { kic after surgery }\end{array}$ & $\begin{array}{l}\text { EZ: } 71 \% \text { intact } \\
\text { preoperatively }\end{array}$ & $61 ; 24 \mathrm{~m}$ & $0.5 / 0.3 \mathrm{~S}$ & $506 / 301 \mathrm{~S}$ \\
\hline 3. Hirano (48) & \multicolumn{2}{|l|}{ Refractory } & $\begin{array}{l}\text { Stable VA if EZ/ ELM } \\
\text { injured }>30 \%\end{array}$ & $\begin{array}{l}\text { 18: }(+) E Z ; \\
12 \mathrm{~m}\end{array}$ & $0.5 / 0.04 \mathrm{~S}$ & $546 / 289 \mathrm{~S}$ \\
\hline 4. Kogo 49) & Refractory & $\begin{array}{l}\text { All rendered pseudopha- } \\
\text { kic after surgery; PRP all }\end{array}$ & $\begin{array}{l}\text { PROS length (EZ ex- } \\
\text { cluded) }\end{array}$ & $36 ; 12 \mathrm{~m}$ & $0.5 / 0.3 \mathrm{~S}$ & $526 / 274 \mathrm{~S}$ \\
\hline 5. Ulrich (50) & Refractory & $\begin{array}{c}\text { Prior macular laser } \\
-61 \%\end{array}$ & $\begin{array}{c}32 \%-E Z / E L M \text { intact: VA } \\
6 / 30 \text { to } 6 / 9\end{array}$ & $31 ; 6 \mathrm{~m}$ & $\begin{array}{c}6 / 25 / / 6 / 15 \\
\mathrm{~S}\end{array}$ & $427 / 357 \mathrm{~S}$ \\
\hline $\begin{array}{l}\text { 6. Browning } \\
\text { (37) }\end{array}$ & $66 \%$-Refr. & $\begin{array}{c}\text { Phaco - } 12 \text { eyes; PRP - in } \\
\text { all. }\end{array}$ & $\begin{array}{c}\text { No relationship of VA } \\
\text { and EZ/ELM }\end{array}$ & $53 ; 12 \mathrm{~m}$ & $\begin{array}{c}6 / 30 / / 6 / 19 \\
\mathrm{~S}\end{array}$ & $505 / 279 \mathrm{~S}$ \\
\hline 7. Iglicki (40) & Naïve & $\mathrm{DDME}<12$ months & $\begin{array}{l}\text { EZ: } 76 \% \text { intact } \\
\text { preoperatively }\end{array}$ & $120 ; 2 y$ & $0.66 / 0.53 \mathrm{~S}$ & $593 / 260 \mathrm{~S}$ \\
\hline $\begin{array}{l}\text { 8. Michalewska } \\
\text { (41) }\end{array}$ & Naïve & $\begin{array}{l}\text { on-tractional-26/44 } \\
\text { eyes; ERM 38\%; } \\
\text { tractional- }\end{array}$ & $\begin{array}{l}\text { No relationship of VA } \\
\text { and EZ/ELM }\end{array}$ & $44 ; 6 m(4-48)$ & $1.35 / 0.77 \mathrm{~S}$ & $595 / 266 \mathrm{~S}$ \\
\hline \multicolumn{7}{|c|}{$\begin{array}{l}\text { S = Statistically significant; phaco- phacoemulsification; CMT- central macular thickness; ERM - central epiretinal membrane; EZ- } \\
\text { ellipsoid zone; }\end{array}$} \\
\hline \multicolumn{7}{|c|}{$\begin{array}{l}\text { ELM- external limiting membrane; ERM- central epiretinal membrane; PROS- photoreceptor outer segment. VA - visual acuity; PDR- } \\
\text { proliferative diabetic retinopathy; PRP - panretinal photpcoagulation; PPV -pars plana vitrectomy; ILM - inner limiting membrane; } \\
\text { CMT - 1-mm central macular thickness. }\end{array}$} \\
\hline
\end{tabular}

Table 1: PPV outcomes in eyes with undetected traction, following preoperative assessment of the foveal layers.

of DDME diagnosis (No.7, Table 1) [40]. Injured outer foveal layers were detected preoperatively in $24 \%$ of eyes when a longer period passed between DDME diagnosis and surgery. Complete macular drying, from mean $593 \mu$ to $260 \mu( \pm 33)$, was achieved already one month following PPV with ILM peeling in all eyes. After two years, all maculae remained dry. Mean BCVA improved from 0.66 to 0.53 logarithm of minimum angle of resolution (logMAR), and $43 \%$ and $32 \%$ gained $\geq 5$ and $\geq 10$ letters, respectively. Central or paracentral macular holes were detected in one eye each. The authors noted that, "for each day PPV is delayed, gaining >5 letters at 24-m dec- reases by 1.8\%". In another study, Michalewska., et al. $(\mathrm{n}=44 ; 17$ with ERM and one with VMT) reported on PPV and ILM peeling that had been conducted within $\leq 12$ months of DDME diagnosis in naïve-treatment eyes (No. 8, Table 1) [41]. Six months postoperatively CMT improved from 595 to $266 \mu \mathrm{m}$, and stabilized until final examination at 8-48 months. BCVA improved (0.3 logMAR) in 55\% of eyes (16\% underwent cataract extraction) and worsened in $2 \%$. Photoreceptorsdefects were detected in $72 \%$ of eyes, which improved to $25 \%$ postoperatively. Macular edema recurred in three (7\%) eyes. 

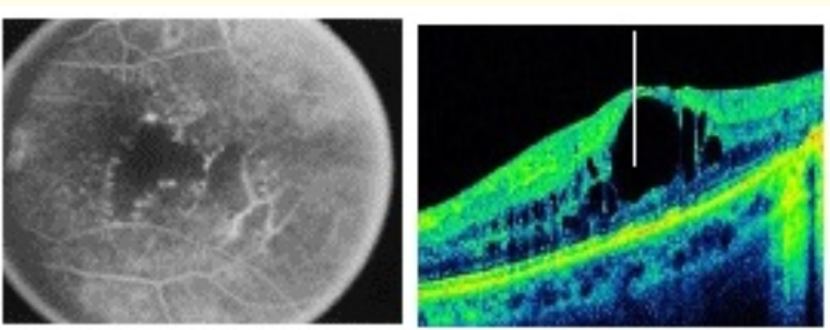

Figure 7: Diabetic macular edema, a large central cyst and macular ischemia. Left: Fluorescein angiogram reveals capillary non-perfusion of $>2$-disc diameters in at least one meridian. Right: The SD-OCT presents a large central macular cyst of $>800 \mu \mathrm{m}$, associated with thinning and disorganization of the inner layers (DRIL).

It is naturally assumed that these eyes that have undergone early-PPV and/or have been naïve-treatment prior to surgery, comprised both the overlooked extrafoveal traction and the vasogenic DDME types. Given the expected high PPV efficacy in eyes with extrafoveal traction, we may conclude that PPV was also very efficacious in the vasogenic DDME eyes. The latter may be explained by the removal of VEGF and pro-inflammatory cytokines and the increased macular oxygenation postoperatively [25].

\section{Modified grid laser photocoagulation for DDME}

The pivotal anti-VEGF DME studies, and thereafter, have typically calculated laser outcomes for both focal DME and mGLP for DDME in one focal/grid group, and without FA in Protocol T, as agreed by the authors $[18,43,44]$. Therefore, conclusions regarding the efficacy of mGLP therapy for a single patient with DDME cannot be drawn from these data. The most common outcomes of mGLP for DDME alone were failures and even worsening of VA and/or CMT $[20,21,45]$. On the other hand, each of the studies has also unveiled eyes that had responded efficaciously and durably to mGLP. However, the DDME pathogenesis in this group of eyes remained enigmatic. For example, in the largest GLP comparative study ( $\mathrm{n}=$ 120), CMT significantly dropped after 12 and 24 months post-GLP, from mean base-line of $379 \mu \mathrm{m}(220-763)$ to $304 \mu \mathrm{m}$ (169-531) and $271 \mu \mathrm{m}$ (156-579), respectively. BCVA improved by $\geq 2$ lines in $30 \%$ of eyes at month- 12 and decreased to $21 \%$ at month-24 [45].
The advent of 3D SD-OCT enabled disclosing the pathogenesis of this enigmatic efficient group. In a mGLP study ( $n=18)$, in addition to eyes with VFT and ERM, DDME-associated extrafoveal traction eyes were also excluded [17]. Eyes with central CNP $\geq 2$ disc diameters (-DD) were likewise excluded, since this dimension of macular ischemia was typically associated with the emergence of a large foveal cyst $(>800 \mu \mathrm{m}$ width) without septae (Figure 7). Such cysts essentially mirrored a DRIL, which was recently described to be related to macular ischemia [3]. After a mean of 15.9 months (4-24) [17], 13 (72.2\%) eyes gradually attained complete drying $(\mathrm{CMT} \leq 290 \mu \mathrm{m})$, while the edema in another eye has dried but partially recurred at month-12 due to emergence of ERM. The causes of the four other mid-term failures were evident: In three eyes, recurrent DDME occurred between months 5 and 9 owing to emergence of extrafoveal vitreous traction that followed incomplete PVD. Edema worsened from base-line in two of these three eyes. Each of these four eyes with recurrent DDME was operable. The remaining eye presented DDME recurrence at month-20, attributed to recent overt macular ischemia, with CNP $>2$-DD. A quantification of clinically significant macular ischemia in required.

Thus, we may deduce that failures associated with mGLP $[20,21,45]$ following exclusion of detected traction membranes, can mainly be referred to overlooking of extrafoveal traction membranes, either prior to mGLP or their emergence following mGLP [17]. As presented above, the prevalence of vasogenic DDME after excluding VFT and ERM was 41\% (and 59\% of extrafoveal traction) [16]. In an attempt to evaluate the mid-term mGLP prognosis while using most current OCTs (rather than 3D SD-OCT): Based on the current data [16,17], durable macular drying by mGLP is expected in $30 \%(41 \times 72.2 \%)$ of DDME eyes, or in one out of three to four eyes. Since most mGLP failures have been related to the presence of overlooked extrafoveal traction, these eyes would potentially be operable following mGLP failure [17]. Similar GLP outcome was achieved in the Pan- American GLP study $(n=120)$ [45].

\section{Strengths and limitations}

To my knowledge, this is the first meta-analysis reporting longterm PPV efficacy in DDME eyes with undetected traction following preoperative assessment of the central macular layers. Improve- 
ment of VA was the rule in all eyes. The focus is also referred for the first time on achieving complete and durable dry maculae.

As well, the two novel pathogeneses enable explanations of these and earlier therapeutic DDME outcomes. Limitations include the relatively few studies as-yet on early-PPV and/or PPV in naïve-treated eyes (total, $>180$ eyes) in eyes with undetected traction $[28,40$ 42]. However, A) the objectivity and accuracy (OCT) of outcomes, and the similar outcomes following PPV under similar conditions (timing of interventions, type of OCT) may strengthen these data; B) during the intravitreal medications era, PPV was commonly performed after many months or years of DME [31]. In contrast, when taut posterior hyaloids have been detected ophthalmoscopically, before the intravitreal medications and OCT eras, PPV was typically conducted after either failed mGLP(s) or in naive-treated eyes [32-34]. The operations most probably typically took place much earlier than during the intravitreal medications era. These surgeries commonly resulted in improvement of VA and mostly in complete and durable macular drying. These outcomes seem to provide additional support for the claim of higher efficacy of early-PPV in naïve-treatment DDME eyes or following minimal interventions, with or without detected traction; C) additional support may be provided from outcomes of delayed PPVs in DME eyes intractable to medication, though following assessments of the foveal layers (Nos. 1-6, Table 1). All 6 studies have resulted in improved VA, but accompanied by only $\sim 50 \%$ (3 studies reported dada) of complete and durable macular drying. Based on the current limited data it is possible that the earlier the surgery with minimal interventions the higher the prevalence of complete and durable macular drying. Further studies would enlighten this important issue. Furthermore, a quantification of the safety level of foveal layers integrity for an efficient PPV is required.

Another limitation regarding early-PPV and/or surgery in naïvetreated eyes is the lack of a comparative study with intravitreal medications. Yet, several PPV studies for eyes with undetected traction have been compared to either tractional ones [5], or to mGLP [26,27], and presented either similar or PPV superiority outcomes, respectively. Until a prospective controlled study is completed, all these data seem to provide a trust of a potential durable superiority of PPV or mGLP over intravitreal medications, which deliver only temporary efficacy and in only $~ 50 \%$ of eyes [19]. Similar limitations are also referred to mGLP outcome in vasogenic DDME [17]. However, the objectivity and accuracy (OCT) of outcomes [17], and the mGLP outcomes for DDME alone after excluding ERM [20], or VFT and ERM [21,45], seem to point for a certain group that would efficaciously respond to mGLP. The fact that the mGLP outcomes had been achieved in conditions of overlooked extrafoveal traction $[20,21,45]$, seems to strengthen the data following their exclusion [17].

\section{Conclusions}

Assessment the integrity of the central macular layers by highresolution OCT has become an important prognostic tool prior to conducting PPV in DME. Enduring macular edema may injure these layers. Studies on early-PPV and/or PPV in treatment-naïve eyes when traction was undetected have reported on very high efficacy in attaining improved VA and complete and long-lasting dry macula. In contrast, delayed PPV in eyes intractable to intravitreal medications aided by mGLP, also resulted in improved VA and edema, but often with residual edema that necessitated continuous, though less frequent treatments. Treatment with mGLP showed durably efficacy in a specific DDME group. When available, 3D SDOCT or en-face 3D-OCT approach, using continuous point-by-point scanning, may help in deciding between early-PPV and mGLP.

Early-PPV and mGLP are also the most cost-effective therapies for DDME. Their highly efficient outcomes may assist in therapeutic decisions, initially at least for populations where budgets or availability of medicationsare a constraint. In such populations, the two therapeutic approaches here described are perhaps their best or only options for saving their eyesight.

\section{Addendum}

In order to detect extrafoveal traction associated with DDME, an OCT with point by point continuous scanning and 3D reconstruction options is highly efficacious. The 3D SD-OCT Topcon 1000 is no longer available on the market. Currently, some swept source (SS)-OCTs do have these options, but their software automatically focus too posteriorly, on the posterior retina/ choroid, resulting in less vitreous gain and poorer 3D outputs. In these, detection of extrafoveal traction is possible by the en-face approach, as described [23].

For the detection of antero-posterior extrafoveal vitreous traction by most current OCT instruments, the following is recommended. A) use a running, continuous OCT scanning, or a software with 
the smallest distance between 2 raster lines; b) increase vitreous gain; c) look for a posterior hyaloid and follow it to its posterior contact site; d) when extrafoveal vitreous traction (rather than adherence) is detected by the B-scan: turn the angle to scanning lines that pass between the oedematose traction site and the foveal oedema, to verify or exclude a continuity between these two oedematose sites (Figure 3); e) use OCT scanning in various fields, or use wide-filed OCT scanning.

Another option to detect extrafoveal traction associated with DDME was presented by Dr. Carl Glittenberg inthe Google. He presented several video clips and described his approach: "This video shows a real-time rendering of an OCT data set of a patient with vitreomacular traction. The data was acquired on a CZM Cirrus HD-OCT 4000 and rendered with a custom plug-in written for MAXON CINEMA 4D. The rendering was done in real-time on a GPU (OpenGL) rendered at $1280 \times 720 \mathrm{p}$. Although the data was acquired on a Cirrus, this rendering program is platform independent and can be achieved with equal quality on an OCT from other compa-

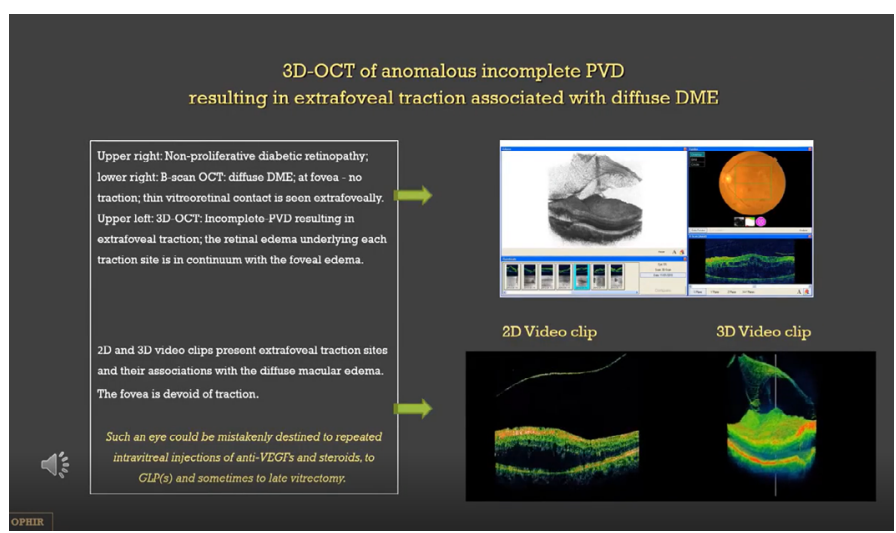

nies (for example OptoVue, Topcon, Heidelberg, etc...)”.

Anomalous incomplete posterior vitreous detachment, extrafoveal traction, and diffuse DME using 3D SD-OCT (audiovisual)

Incomplete posterior vitreous detachment resulting in extrafoveal vitreous traction. At the fovea (marked by a vertical line) no traction is detected. The 2D video clip illustrates point-by-point continuous scanning, presenting extrafoveal traction sites and their continuity with the diffuse DME. The 3D-OCT video clip enables perception of these extrafoveal traction membranes and their associations with the DDME. The retinal edema underlying the traction site is in a continuum with the foveal edema. Comment: Using most current OCTs, such an eye could be mistakenly considered non-tractional and destined to receive repeated injections of anti-VEGFs, steroids, grid laser photocoagulation, and sometimes late vitrectomy. Recent studies suggest a consideration of early-PPV in DDME eyes.

\section{Conflict of Interest}

The author declares on no conflict of interest

\section{Bibliography}

1. Sadda SR., et al. "Relationship between duration and extent of edema and visual acuity outcome with ranibizumab in diabetic macular edema: A post hoc analysis of Protocol I data". Eye 34 (2020): 480-490.

2. Sakamoto A., et al. "Association between foveal photoreceptor status and visual acuity after resolution of diabetic macular edema by pars plana vitrectomy". Graefe's Archive for Clinical and Experimental Ophthalmology 247 (2009): 1325-1330.

3. Cennamo G., et al. "Evaluation of vessel density in disorganization of retinal inner layers after resolved diabetic macular edema using optical coherence tomography". PLoS One 16.1 (2021): 0244789.

4. Schmidt-Erfurth U., et al. "Guidelines for the Management of Diabetic Macular Edema by the European Society of Retina Specialists (EURETINA)”. Ophthalmologica 237 (2017): 185222.

5. Bonnin S., et al. "Vitrectomy with internal limiting membrane peeling for tractional and nontractional diabetic macular edema. Long-term results of a comparative study". Retina 35 (2015): 921-928.

6. Gandorfer A., et al. "Epiretinal pathology of diffuse diabetic macular edema associated with vitreomacular traction". American Journal of Ophthalmology 139 (2005): 638-652. 
7. Elman MJ., et al. "Randomized trial evaluating ranibizumab plus prompt or deferred laser or triamcinolone plus prompt laser for diabetic macular edema". Ophthalmology 117 (2010): 1064-1077.

8. Mitchell P., et al. "The RESTORE Study: Ranibizumab monotherapy or combined with laser versus laser monotherapy for diabetic macular edema”. Ophthalmology 118 (2011): 615-625.

9. Elman MJ., et al. "Expanded 2-year follow-up of ranibizumab plus prompt or deferred laser or triamcinolone plus prompt laser for diabetic macular edema”. Ophthalmology 118 (2011): 609-614.

10. Wells JA., et al. "Aflibercept, bevacizumab, or ranibizumab for diabetic macular edema: two year results from a comparative effectiveness randomized clinical trial". Ophthalmology 123 (2016): 1351-1359.

11. Ciulla TA., et al. "Real-world outcomes of anti- vascular endothelial growth factor therapy in diabetic macular edema in the United States". Ophthalmology Retina 2 (2018): 1179-1187.

12. Egan C., et al. "The United Kingdom diabetic retinopathy electronic medical record users group, Report 1: Baseline characteristics and visual acuity outcomes in eyes treated with intravitreal injections of ranibizumab for diabetic macular edema". British Journal of Ophthalmology 101 (2017): 75-78.

13. Ciulla A., et al. "Visual acuity outcomes and anti-VEGF therapy intensity in diabetic macular edema: a real-world analysis of 28,658 patient eyes". British Journal of Ophthalmology (2020).

14. Glassman AR., et al. "Five-Year Outcomes after initial Aflibercept, Bevacizumab, or Ranibizumab treatment for diabetic macular edema (Protocol T Extension Study)". Ophthalmology 127 (2020): 1201-1210.

15. Ophir A., et al. "Extrafoveal vitreous traction associated with diabetic diffuse macular edema". Eye (Lond) 24 (2010): 347-353.

16. Ophir A., et al. "Vitreous traction and epiretinal membranes in diabetic macular edema using spectral-domain optical coherence tomography". Eye (Lond) 24 (2010): 1545-1553.
17. Ophir A., et al. "Importance of 3-D image reconstruction of spectral-domain OCT on outcome of grid laser photocoagulation for diffuse diabetic macular edema". International Journal of Ophthalmology 6 (2013): 836-843.

18. Ophir A. "Full-field 3-D optical coherence tomography imaging and treatment decision in diffuse diabetic macular edema". Investigative Ophthalmology and Visual Science 55 (2014): 30523053.

19. Ophir A. Correspondence: "Early and long-term responses to anti-vascular endothelial growth factor therapy in diabetic macular edema: Analysis of protocol I data". American Journal of Ophthalmology 177 (2017): Reply: 230-231.

20. Lee CM and Olk RJ. "Modified grid laser photocoagulation for diffuse DME. Long-term visual results". Ophthalmology 98 (1991): 1594-1602.

21. Yanyali A., et al. "Pars plana vitrectomy and removal of the internal limiting membrane in diabetic macular edema unresponsive to grid laser photocoagulation". European Journal of Ophthalmology 16 (2006): 573-81.

22. Fatima N., et al. "Frequency of vitreomacular traction in diabetic macular edema on optical coherence tomography". Pakistan Armed Forces Medical Journal 67 (2017): 47-50.

23. Adhi M., et al. "Three-dimensional enhanced imaging of vitreoretinal interface in diabetic retinopathy using swept-source optical coherence tomography". American Journal of Ophthalmology 162 (2016): 140-149.

24. Kashani AH., et al. "Impaired retinal perfusion resulting from vitreoretinal traction: A mechanism of retinal vascular insufficiency". Ophthalmic Surgery, Lasers and Imaging Retina 47 (2016): 1-11.

25. Stefansson E., et al. "Increased retinal oxygen supply following pan-retinal photocoagulation and vitrectomy and lensectomy". Transactions of the American Ophthalmological Society 79 (1981): 307-334.

26. Adelman R., et al. "Strategy for the Management of Diabetic Macular Edema: The European Vitreo-Retinal Society Macular Edema Study". BioMed Research International (2015). 
27. Yanyali A., et al. "Modified grid laser photocoagulation versus pars plana vitrectomy with internal limiting membrane removal in diabetic macular edema". American Journal of Ophthalmology 139 (2005): 795-801.

28. Hagenau F., et al. "Vitrectomy for diabetic macular edema: Optical coherence tomography criteria and pathology of the vitreomacular interface". American Journal of Ophthalmology 200 (2019): 34-46.

29. Sebag J. "Vitreoschisis". Graefe's Archive for Clinical and Experimental Ophthalmology 246 (2008): 329-332.

30. Ophir A and Martinez MR. "Epiretinal membranes and incomplete posterior vitreous detachment in diabetic macular edema, detected by spectral-domain optical coherence tomography". Investigative Ophthalmology and Visual Science 52 (2011): 6414-6420.

31. Flaxel CJ., et al. "Factors associated with visual acuity outcomes after vitrectomy for diabetic macular edema: Diabetic Retinopathy Clinical Research network". Retina 30 (2010): 14881495.

32. Lewis H., et al. "Vitrectomy for diabetic macular traction and edema associated with posterior hyaloidal traction". Ophthalmology 99 (1992): 753-759.

33. Pendergast SD., et al. "Vitrectomy for diffuse diabetic macular edema associated with a taut premacular posterior hyaloid". American Journal of Ophthalmology 130 (2000): 178-186.

34. Gandorfer A., et al. "Resolution of diabetic macular edema after surgical removal of the posterior hyaloid and the inner limiting membrane". Retina 20 (2000): 126-133.

35. Landers MB III., et al. "Early vitrectomy for DME: Does it have a role?" Retin Physician 10 (2013): 46-53.

36. Schmidt-Erfurth U., et al. "Three-dimensional ultrahigh- resolution optical coherence tomography of macular diseases". Investigative Ophthalmology and Visual Science 46 (2005): 33933402 .

37. Browning DJ., et al. "Vitrectomy for center-involved diabetic macular edema”. Clinic Ophthalmology 10 (2016): 735-742.
38. Kroll P., et al. "Vitreopapillary traction in proliferative diabetic vitreoretinopathy". British Journal of Ophthalmology 83 (1999): 261-264.

39. Yanyali A., et al. "Quantitative assessment of photoreceptor layer in eyes with resolved edema after pars plana vitrectomy with internal limiting membrane removal for diabetic macular edema”. Ophthalmologica 226 (2011): 57-63.

40. Iglicki M., et al. "Biomarkers and predictors for functional and anatomic outcomes for small gauge pars plana vitrectomy and peeling of the internal limiting membrane in naïve diabetic macular edema: The VITAL Study". PloS One 13.7 (2018).

41. Michalewska Z., et al. "Vitrectomy in the management of diabetic macular edema in treatment-naïve patients". Canadian Journal of Ophthalmology 53 (2018): 402-407.

42. La Haij EC., et al. "Vitrectomy results in diabetic macular edema without evident vitreomacular traction". Graefe's Archive for Clinical and Experimental Ophthalmology 239 (2001): 264-70.

43. Shanmugam MP., et al. Correspondence: "Aflibercept, bevacizumab, or ranibizumab for diabetic macular edema: two-year results from a comparative effectiveness randomized clinical trial". Ophthalmology 124 (2017): e5.

44. Wells JA., et al. Reply: "Aflibercept, bevacizumab, or ranibizumab for diabetic macular edema: two-year results from a comparative effectiveness randomized clinical trial". Ophthalmology 124 (2017): e5-6.

45. Arevalo JF., et al. "Intravitreal bevacizumab plus grid laser photocoagulation or intravitreal bevacizumab or grid laser photocoagulation for diffuse diabetic macular edema". Retina 33 (2013): 403-413.

46. Uji A., et al. "Influence of vitrectomy surgery on the integrity of outer retinal layers in diabetic macular edema". Retina 38 (2018): 163-172.

47. Miyamoto N., et al. "Restoration of photoreceptor outer segments up to 24 months after pars plana vitrectomy in patients with diabetic macular edema". Ophthalmology Retina (2017): 1-6. 
48. Hirano T and Murata T. "Vitrectomy for DME without macular traction". Retin Physician 10 (2013): 61-64.

49. Kogo J., et al. "Foveal microstructure analysis in eyes with diabetic macular edema treated with vitrectomy". Advances in Therapy 34 (2017): 2139-2149.

50. Ulrich JN. “Pars Plana Vitrectomy with Internal Limiting Membrane Peeling for nontractional diabetic macular edema”. The Open Ophthalmology Journal 11 (2017): 5-10.

\section{Assets from publication with us}

- Prompt Acknowledgement after receiving the article

- Thorough Double blinded peer review

- Rapid Publication

- Issue of Publication Certificate

- High visibility of your Published work

Website: www.actascientific.com/

Submit Article: www.actascientific.com/submission.php

Email us: editor@actascientific.com

Contact us: +919182824667 